\title{
DILATIONS AND SUBNORMALITY
}

\author{
WACLAW SZYMANSKI
}

AbStract. A general view of subnormality via the dilation theory is presented.

1. Introduction. In [13] Sz.-Nagy observed that the well-known Halmos positivity condition for subnormality of a Hilbert space operator means that a certain function is positive definite on a certain $*$-semigroup. This way subnormality of one operator became a part of the general dilation theory. Since then new positivity conditions for one operator, and for families of operators to be subnormal have been introduced by Embry [7] and Lubin [10], respectively. In this paper we shall show that Sz.-Nagy's idea carries over to arbitrary semigroups of operators providing a unified picture of subnormality which contains Halmos's [8] and Bram's [4] as well as Ito's [9] results. Moreover, we shall find a place in this picture for Embry's and Lubin's conditions. The latter ones are more subtle from the dilation theory standpoint - one has to use a dilation theorem for semigroups [14], not only for $*$-semigroups. We should like to point out that, unlike the approaches taken up by all authors dealing with subnormality of more than one operator, our approach is totally "lifting-of-the-commutant-free." The "lifting of the commutant" problem always involves a "boundedness condition." As it has been proved by Bram [4] and simplified by Szafraniec [12] for one operator, no boundedness condition is needed. We shall show that not only is this true for the general case, but also can be seen by.dilation methods, different from and simpler than Bram's methods.

2. Preliminaries. Let $S$ be a semigroup with unit. Let $H$ be a Hilbert space. $B(H)$ denotes the multiplicative semigroup of all linear bounded operators in $H$. $F(S, H)$ stands for the family of all functions from $S$ to $H$ vanishing off a finite subset of $S$. A function $A: S \times S \rightarrow B(H)$ is called positive definite (PD) if

$$
\sum(A(s, t) f(s), f(t)) \geqslant 0
$$

for each $f \in F(S, H)$. A triple $(K, \pi, R)$ is called a dilation of a function $A$ : $S \times S \rightarrow B(H)$ if $K$ is a Hilbert space, $\pi: S \rightarrow B(K)$ is a semigroup homomorphism, $R: H \rightarrow K$ is linear and bounded, and $A(s, t)=R^{*} \pi(t)^{*} \pi(s) R, s, t \in S$.

Received by the editors July 1, 1986.

1980 Mathematics Subject Classification (1985 Revision). Primary 47D05, 43A35, 47A20, 47B20.

Ke' nords and phrases. Positive definite function, dilation, *-dilation, subnormality, quasinormality. 
The condition $K=$ the closed linear span of $\pi(S) R H$ determines a dilation $(K, \pi, R)$ of $A$ uniquely up to a unitary isomorphism. Such a dilation is called minimal. The following useful proposition follows immediately from the minimality condition.

(2.1) Proposition. Let $(K, \pi, R)$ be the minimal dilation of $A: S \times S \rightarrow B(H)$. Let $u, u^{\prime}, v, v^{\prime} \in S$. Then $\pi(v)^{*} \pi(u)=\pi\left(v^{\prime}\right)^{*} \pi\left(u^{\prime}\right)$ if and only if $A(u s, v t)=$ $A\left(u^{\prime} s, v^{\prime} t\right)$ for all $s, t \in S$.

The most general dilation theorem we shall need here is the following consequence of Corollary 3.18 in [14], and of Proposition (2.1).

(2.2) TheOrem. Let $S$ be a semigroup with unit. Let $A: S \times S \rightarrow B(H)$ be a function. Suppose there is a function $d: S \rightarrow S$ such that $A(u s, u t)=A(d(u) s, t)$, $u, s, t \in S$. Then $A$ has a dilation if and only if $A$ is $P D$ and there are real functions $b$ on $H \times H$ and $c$ on $S$ such that $c(s t) \leqslant c(s) c(t), s, t \in S$, and $|(A(s, t) x, y)| \leqslant$ $b(x, y) c(s) c(t), x, y \in H, s, t \in S$. If $(K, \pi, R)$ is the minimal dilation of $A$, then $\pi(u)^{*} \pi(u)=\pi(d(u)), u \in S$.

A semigroup $S$ with unit 1 is called a *-semigroup if a mapping $*: S \rightarrow S$ is defined so that $(s t)^{*}=t^{*} s^{*},\left(s^{*}\right)^{*}=s, s, t \in S, 1^{*}=1$.

Let $S$ be a $*$-semigroup. A semigroup homomorphism $\pi: S \rightarrow B(H)$ is called a *-homomorphism if $\pi\left(s^{*}\right)=\pi(s)^{*}, s \in S$. A dilation $(K, \pi, R)$ of a function $A$ : $S \times S \rightarrow B(H)$ is called a $*$-dilation if $\pi$ is a $*$-homomorphism.

(2.3) Corollary. Let $S$ be a *-semigroup. A function $A: S \times S \rightarrow B(H)$ has a *-dilation if and only if $A$ has a dilation and $A\left(u^{*} s, t\right)=A(s, u t), u, s, t \in S$.

This corollary, whose proof follows from Proposition (2.1), is a version of the Sz.-Nagy dilation theorem for *-semigroups (cf. [13, Principal Theorem; 2, *-Dilation Theorem; 14, Proposition 4.1.1]).

Let $S$ be a semigroup with unit. A semigroup homomorphism $\pi: S \rightarrow B(H)$ will be called normal if $\pi(t)^{*} \pi(s)=\pi(s) \pi(t)^{*}, s, t \in S$, quasinormal if $\pi(t)^{*} \pi(t) \pi(s)$ $=\pi(s) \pi(t)^{*} \pi(t)$ and $\pi(t) \pi(s)=\pi(s) \pi(t), s, t \in S$, and subnormal if there is a Hilbert space $K$ containing $H$ and a normal homomorphism $\tau: S \rightarrow B(H)$ such that $H$ is invariant for each $\tau(s)$, and $\tau(s) \mid H=\pi(s), s \in S$. Such $\tau$ will be called a normal extension of $\pi$. If $K$ equals the closed linear span of $\tau(S)^{*} H$, such an extension will be called minimal. The minimality condition determines the normal extension uniquely up to a unitary isomorphism. $N$ denotes the additive semigroup of all nonnegative integers, $N^{n}$ is the $n$-times product semigroup. Commuting operators $T_{1}, \ldots, T_{n}$ in $B(H)$ are called a subnormal $n$-tuple if the homomorphism $\pi: \quad N^{n} \rightarrow B(H), \pi\left(i_{1}, \ldots, i_{n}\right)=T_{1}^{i_{1}} \cdots T_{n}^{i_{n}}$, is subnormal. Its minimal normal extension $\tau: N^{n} \rightarrow B(K)$ has the form $\tau\left(i_{1}, \ldots, i_{n}\right)=N_{1}^{i_{1}} \cdots N_{n}^{i_{n}}$, where $N_{1}, \ldots, N_{n}$ are commuting normal operators in $K$; they are called a common normal extension of $T_{1}, \ldots, T_{n}$. In [10] a family $\Gamma \subset B(H)$ was called jointly quasinormal if $C D^{*} D=$ $D^{*} D C, C D=D C, C, D \in \Gamma$. It is plain that if $\Gamma$ is jointly quasinormal, then so is the multiplicative semigroup it generates. Therefore we may restrict attention to quasinormal semigroups. By Fuglede's theorem, if $\pi: S \rightarrow B(H)$ is a normal 
homomorphism, then $\pi(s), s \in S$, are normal mutually commuting operators, no matter whether $S$ is commutative or not. Therefore, if $\pi: S \rightarrow B(H)$ is a subnormal homomorphism, then $\pi(s), s \in S$, are commuting subnormal operators.

3. The results. Let $S$ be an arbitrary semigroup with unit 1 . Elements of $S \times S$ will be denoted by $s_{0}=\left(s, s^{\prime}\right), t_{0}=\left(t, t^{\prime}\right)$, etc., $s, s^{\prime}, t, t^{\prime} \in S$. In $S \times S$ define

an algebraic operation: $s_{0} t_{0}=\left(s t, s^{\prime} t^{\prime}\right), s_{0}=\left(s, s^{\prime}\right), t_{0}=\left(t, t^{\prime}\right) \in S \times S$,

an involution: $s_{0}^{*}=\left(s^{\prime}, s\right), s_{0}=\left(s, s^{\prime}\right) \in S \times S$.

(In case $S=N$ the above operation and involution were introduced in [13].) With this operation and this involution $S \times S$ becomes a $*$-semigroup with unit $(1,1)$. This *-semigroup will be denoted by $S_{0} . S$ itself identifies with a subsemigroup of $S_{0}$ by the mapping $s \rightarrow(s, 1), s \in S$, which is a one-to-one semigroup homomorphism. In what follows $S$ will be treated as a subsemigroup of $S_{0}$ and we shall write $s$ instead of $(s, 1), s \in S$. If $S$ is an arbitrary semigroup, $S_{0}$ does not need to be commutative. The above operation, however, has the following simple, but crucial property:

$$
\left.t^{*} s=s t^{*}, \quad s, t \in S \quad \text { (i.e. }(1, t)(s, 1)=(s, 1)(1, t)=(s, t)\right) .
$$

This property, which implies also that each element of $S_{0}$ can be uniquely written in the form $t^{*} s, s, t \in S$, proves immediately the following characterization of *-homomorphisms of $S_{0}$.

(3.2) Proposition. (a) If $\tau_{0}: S_{0} \rightarrow B(H)$ is a*-homomorphism, then $\tau=\tau_{0} \mid S$ : $S \rightarrow B(H)$ is a normal homomorphism such that $\tau_{0}\left(s_{0}\right)=\tau\left(s^{\prime}\right)^{*} \tau(s)$ for each $s_{0}=$ $\left(s, s^{\prime}\right) \in S_{0}$.

(b) If $\tau: S \rightarrow B(H)$ is a normal homomorphism, then $\tau_{0}: S_{0} \rightarrow B(H)$ defined by $\tau_{0}\left(s_{0}\right)=\tau\left(s^{\prime}\right)^{*} \tau(s), s_{0}=\left(s, s^{\prime}\right) \in S_{0}$ is a*-homomorphism, and $\tau=\tau_{0} \mid S$.

Now define $S_{d}=\{(u s, u): u, s \in S\}=\left\{u^{*} u s: u, s \in S\right\}$. Clearly, $S \subset S_{d} \subset S_{0}$. If $S=N$, this subset consists of all lattice points between the "lines" $S$ and $\{(u, u)$ : $u \in S\}$ in the upper right corner of the plane. When dealing with $S_{d}$ it will be assumed that $S$ is commutative. Then $S_{d}$ is a subsemigroup of $S_{0}$, which is not a *-semigroup with the above defined involution. The reason why $S_{d}$ is important is given in the following

(3.3) Proposition. (a) If $\tau: S_{d} \rightarrow B(H)$ is a semigroup homomorphism such that $\tau\left(u^{*} u\right)=\tau(u)^{*} \tau(u), u \in S$, then $\sigma=\tau \mid S: S \rightarrow B(H)$ is a quasinormal homomorphism and $\tau\left(u^{*} u s\right)=\sigma(u)^{*} \sigma(u) \sigma(s), u, s \in S$.

(b) If $\sigma: S \rightarrow B(H)$ is a quasinormal homomorphism, then $\tau: S_{d} \rightarrow B(H)$ defined by $\tau\left(u^{*} u s\right)=\sigma(u)^{*} \sigma(u) \sigma(s), s, u \in S$, is a semigroup homomorphism such that $\tau\left(u^{*} u\right)=\tau(u)^{*} \tau(u), u \in S$.

One proves this proposition by a straightforward calculation. Now we shall formulate two conditions. The first one (HB) in the case of a single operator $(S=N)$ is the famous Halmos condition [8] which was proved by Bram [4] to characterize subnormal operators. The second one (EL) was first introduced by Embry [7] for one operator, and then by Lubin [10] for families of commuting 
operators. Let $\pi: S \rightarrow B(H)$ be a semigroup homomorphism. We shall say that $\pi$ satisfies the condition

$$
\begin{aligned}
& \text { if } \sum(\pi(s) f(t), \pi(t) f(s)) \geqslant 0 \quad \text { for each } f \in F(S, H), \\
& \text { if } \sum(\pi(s t) f(t), \pi(s t) f(s)) \geqslant 0 \quad \text { for each } f \in F(S, H) .
\end{aligned}
$$

Now we are ready to state the main theorem.

(3.4) TheOREM. Let $S$ be a semigroup with unit. Let $S_{0}, S_{d}$ be as defined above. Let $\pi: S \rightarrow B(H)$ be a semigroup homomorphism. Define $\Phi: S_{0} \rightarrow B(H)$ by $\Phi\left(s_{0}\right)=$ $\pi\left(s^{\prime}\right)^{*} \pi(s), \quad s_{0}=\left(s, s^{\prime}\right) \in S_{0}$, and $A: S_{0} \times S_{0} \rightarrow B(H)$ by $A\left(s_{0}, t_{0}\right)=\Phi\left(t_{0}^{*} s_{0}\right)$, $s_{0}, t_{0} \in S_{0}$. Then the following conditions are equivalent:

(a) $\pi$ is subnormal,

(b) A has a *-dilation,

(c) $A$ is $P D$ on $S_{0}$,

(d) the operators $\pi(s), s \in S$, commute, and $\pi$ satisfies (HB),

(e) for each $n \in N, s_{1}, \ldots, s_{n} \in S$, the operators $\pi\left(s_{1}\right), \ldots, \pi\left(s_{n}\right)$ are a subnormal n-tuple,

(f) there is a Hilbert space $K_{1}$ containing $H$ and a quasinormal homomorphism $\tau_{1}$ : $S \rightarrow B\left(K_{1}\right)$ such that $H$ is invariant for each operator $\tau_{1}(s), s \in S$, and $\pi(s)=$ $\tau_{1}(s) \mid H, s \in S$.

If $S$ is a commutative semigroup, then each of the above conditions is equivalent to each of the following ones:

(g) The function $A_{d}: S_{d} \times S_{d} \rightarrow B(H)$ defined by $A_{d}=A \mid S_{d} \times S_{d}$ has a dilation,

(h) $A_{d}$ is $P D$ on $S_{d}$,

(i) $\pi$ satisfies (EL).

Moreover, (a) and (b) are related as follows: If $(K, \tau, R)$ is a (the minimal, resp.) *-dilation of $A$, then $R$ is an isometric embedding of $H$ into $K, H$ identified with its image under $R$ is invariant for $\tau(s), s \in S$, and $\tau \mid S: S \rightarrow B(K)$ is a (the minimal, resp.) normal extension of $\pi$. Conversely, if $\tau_{0}: S \rightarrow B(K)$ is a (the minimal, resp.) normal extension of $\pi$, if $R: H \rightarrow K$ is the inclusion map, and if $\tau: S_{0} \rightarrow B(K)$ is defined by $\tau\left(s_{0}\right)=\tau_{0}\left(s^{\prime}\right) \tau_{0}(s), s_{0}=\left(s, s^{\prime}\right) \in S$, then $(K, \tau, R)$ is a the minimal, resp.) *-dilation of $A$. A similar relationship holds between (f) and (g).

Proof. The theorem will be proved according to the following chart of implications:

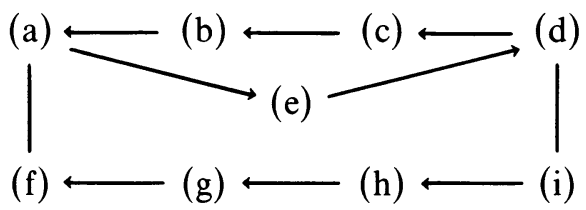

of which (a) $\rightarrow(\mathrm{e})$ is clear.

(d) $\rightarrow$ (i) If $f \in F(S, H)$, then $g: S \rightarrow H$ defined by $g(u)=\pi(u) f(u), u \in S$, also belongs to $F(S, H)$. Hence

$$
\sum(\pi(s t) f(t), \pi(s t) f(s))=\sum(\pi(s) g(t), \pi(t) g(s)) \geqslant 0 .
$$


(e) $\rightarrow$ (d) Take $n \in N, s_{1}, \ldots, s_{n} \in S, x_{1}, \ldots, x_{n} \in H$, and a common normal extension $N_{1}, \ldots, N_{n}$ of $\pi\left(s_{1}\right), \ldots, \pi\left(s_{n}\right)$. Then

$$
\sum\left(\pi\left(s_{i}\right) x_{j}, \pi\left(s_{j}\right) x_{i}\right)=\left\|\sum N_{j}^{*} x_{j}\right\|^{2} \geqslant 0 .
$$

Thus $\pi$ satisfies (HB).

$(\mathrm{g}) \rightarrow$ (f) Notice first that

$$
A\left(s_{0}, t_{0}\right)=\Phi\left(t_{0}^{*} s_{0}\right)=\pi\left(s^{\prime}\right)^{*} \pi(t)^{*} \pi\left(t^{\prime}\right) \pi(s)
$$

for each $s_{0}=\left(s, s^{\prime}\right), t_{0}=\left(t, t^{\prime}\right) \in S_{0}$. Let $(K, \tau, R)$ be the minimal dilation of $A_{d}$. Take $s_{0}=u^{*} u s=(u s, u) \in S_{d}$. Then $(3.5)$ with $t_{0}=(1,1)$ gives

$$
\pi(u)^{*} \pi(u s)=A\left(s_{0}, 1\right)=R^{*} \tau(u)^{*} \tau(u s) R, \quad u, s \in S .
$$

If $u=s=1$, then $R^{*} R=I_{H}$. Thus $H$ can be treated as a subspace of $K$, and $P=R^{*}$ as a projection of $K$ onto $H$. The above equality takes the form

$$
\pi(u)^{*} \pi(u s)=P \tau(u)^{*} \tau(u s) \mid H, \quad u, s \in S .
$$

If $s=1$, it follows from a standard observation (see, e.g., [13]) that $H$ is invariant for $\tau(u)$, and $\tau(u) \mid H=\pi(u), u \in S$. Moreover, if $u \in S$, then

$$
A_{d}\left(u^{*} u s, t\right)=A\left(u^{*} u s, t\right)=\Phi\left(t^{*} u^{*} u s\right)=A(u s, u t)=A_{d}(u s, u t)
$$

for all $s, t \in S$. By Proposition (2.1), $\tau\left(u^{*} u\right)=\tau(u)^{*} \tau(u), u \in S$. By Proposition (3.3)(a), $\tau$ is quasinormal.

(b) $\rightarrow$ (a) Let $(K, \tau, R)$ be the minimal *-dilation of $A$. The proof repeats the above arguments using Proposition (3.2)(a) instead of (3.3)(a), and dropping the next to last step that uses Proposition (2.1) as unnecessary.

(c) $\rightarrow$ (b) Define $c\left(s_{0}\right)=\|\pi(s)\|\left\|\pi\left(s^{\prime}\right)\right\|, s_{0}=\left(s, s^{\prime}\right) \in S_{0}$, and $b(x, y)=\|x\|\|y\|$, $x, y \in H$. It is clear that $b, c$ satisfy the assumptions of Theorem (2.2). Moreover, $A\left(u_{0}^{*} s_{0}, t_{0}\right)=A\left(s_{0}, u_{0} t_{0}\right), u_{0}, s_{0}, t_{0} \in S_{0}$. Therefore, by Theorem (2.2) with $d\left(u_{0}\right)=$ $u_{0}^{*} u_{0}, u_{0} \in S_{0}$, and Corollary (2.3), $A$ has a $*$-dilation. (To prove this. implication it would be sufficient to use the dilation theorem for $*$-semigroups (see, e.g., [13 or 2 , *-Dilation Theorem]). The full strength of Theorem (2.2) is needed to prove the next one.)

(h) $\rightarrow$ (g) Firstly notice that $u_{0}^{*} u_{0} \in S_{d}$ for each $u_{0} \in S_{0}$, and that $A_{d}\left(u_{0}^{*} u_{0} s_{0}, t_{0}\right)$ $=A_{d}\left(u_{0} s_{0}, u_{0} t_{0}\right), u_{0}, s_{0}, t_{0} \in S_{d}$. Then apply Theorem (2.2) to the function $A_{d}$ with the above defined $b, c$, and $d \mid S_{d}$.

(d) $\rightarrow$ (c) This proof follows essentially the Sz.-Nagy arguments [13] for one operator. Take $f \in F\left(S_{0}, H\right)$. Define $g(s)=\sum_{v}, \pi(v) f(s, v), s \in S$. Then $g \in$ $F(S, H)$ and since the operators $\pi(s), s \in S$, commute, (3.5) implies that

$$
\begin{aligned}
\sum\left(A\left(s_{0}, t_{0}\right) f\left(s_{0}\right), f\left(t_{0}\right)\right) & =\sum\left(\pi\left(t^{\prime}\right) \pi(s) f\left(s, s^{\prime}\right), \pi(t) \pi\left(s^{\prime}\right) f\left(t, t^{\prime}\right)\right) \\
& =\sum\left(\pi\left(t^{\prime}\right) g\left(s^{\prime}\right), \pi\left(s^{\prime}\right) g\left(t^{\prime}\right)\right) \geqslant 0 .
\end{aligned}
$$

Thus $A$ is PD.

(i) $\rightarrow$ (h) Take $f \in F\left(S_{d}, H\right)$. Define $g(u)=\sum_{s} \pi(s) f(u s, u), u \in S$. Then $g \in$ $F(S, H)$. If $s, t, u, v \in S$, then $s_{0}=u^{*} u s=(u s, u), t_{0}=v^{*} v t=(v t, v)$ belong to $S_{d}$, and, by (3.5),

$$
A_{d}\left(s_{0}, t_{0}\right)=\pi(u)^{*} \pi(t)^{*} \pi(v)^{*} \pi(v) \pi(u) \pi(s)
$$


Hence

$$
\begin{aligned}
\sum( & \left.A_{d}\left(s_{0}, t_{0}\right) f\left(s_{0}\right), f\left(t_{0}\right)\right) \\
& =\sum(\pi(v) \pi(u) \pi(s) f(u s, u), \pi(v) \pi(t) \pi(u) f(v t, v)) \\
& =\sum(\pi(v u) g(u), \pi(v u) g(v)) \geqslant 0 .
\end{aligned}
$$

Thus $A_{d}$ is PD. The relationship between the minimality conditions in the last two assertions of the theorem follows from Fuglede's theorem.

(f) $\rightarrow$ (a) will be a consequence of Theorem (3.10). Q.E.D.

To finish the above proof immediately we could quote Theorem 4.5 of [10]. Its proof, however, consists of combining a transfinite induction argument with Theorem 5 of [15]. Let us point out that the method originated by Bram in his proof of Theorem 7 in [4], which describes the "lifting of the commutant" effect, is the cornerstone of the proofs of these two theorems as well as of various other ones concerning the existence of a (quasi) normal extension (cf., e.g., proofs of Theorem 1 of [15], Lemma 4.1 of [10]). This approach disagrees with the general principle of this paper to remain "lifting-of-the-commutant-free." Therefore a more straightforward proof will be given, which also provides us with a better understanding of the relationship between quasinormality and subnormality. The equivalence of (a) and (e) in Theorem (3.4) is essentially Ito's Theorem 1 of [9]. Ito's proof again uses Bram's method, so it is entirely different from the one given above. The implication (d) $\rightarrow$ (c) is proved for $S=N$ in [13]. (c) $\rightarrow$ (b) for $S=N$ can be found in [12]. Some assertions of this theorem for $S=N$ are in [5, Chapter 3, Theorem 1.9]. A part of this theorem for $S=N^{n}$ can be found in [3]. Notice that the conditions (a)-(e) of Theorem (3.4) have already been proved to be equivalent.

(3.6) Corollary. Let $S, S^{\prime}$ be semigroups with units. Let $\pi: S \rightarrow B(H), \pi^{\prime}$ : $S^{\prime} \rightarrow B(H)$ be semigroup homomorphisms. Define a homomorphism $\pi \pi^{\prime}$ on the product semigroup $S \times S^{\prime}$ by $\pi \pi^{\prime}\left(s, s^{\prime}\right)=\pi(s) \pi\left(s^{\prime}\right), s \in S, s^{\prime} \in S^{\prime}$.

(a) If all $\pi^{\prime}\left(s^{\prime}\right)$ commute with all $\pi(s), s \in S, s^{\prime} \in S^{\prime}, \pi$ is normal, and $\pi^{\prime}$ is subnormal, then $\pi \pi^{\prime}$ is subnormal.

(b) $\pi \pi^{\prime}$ is subnormal if and only if $\pi$ is subnormal (with the minimal normal extension $\tau: S \rightarrow B(K)$ ) and there is a subnormal homomorphism $\tau^{\prime}: S^{\prime} \rightarrow B(K)$ such that $H$ is invariant for each $\tau^{\prime}\left(s^{\prime}\right), \tau^{\prime}\left(s^{\prime}\right) \mid H=\pi^{\prime}\left(s^{\prime}\right)$, and all $\tau(s)$ commute with all $\tau^{\prime}\left(s^{\prime}\right), s \in S, s^{\prime} \in S^{\prime}$.

Proof. In case $S=S^{\prime}=N$, (a) seems to be a folklore-type result, whose proof was given in [6, Proposition 2.9]. Here is the proof of (a) in the general case, equally short and simple. Take $f \in F\left(S \times S^{\prime}, H\right)$. Define $h\left(u^{\prime}\right)=\sum_{v \in S} \pi(v)^{*} f\left(v, u^{\prime}\right), u^{\prime} \in$ $S^{\prime}$. Then $h \in F\left(S^{\prime}, H\right)$, and

$$
\begin{aligned}
\sum\left(\pi \pi^{\prime}\left(s, s^{\prime}\right)\right. & \left.f\left(t, t^{\prime}\right), \pi \pi^{\prime}\left(t, t^{\prime}\right) f\left(s, s^{\prime}\right)\right) \\
= & \sum\left(\pi^{\prime}\left(s^{\prime}\right) \pi(t)^{*} f\left(t, t^{\prime}\right), \pi^{\prime}\left(t^{\prime}\right) \pi(s)^{*} f\left(s, s^{\prime}\right)\right) \\
= & \sum\left(\pi^{\prime}\left(s^{\prime}\right) h\left(t^{\prime}\right), \pi^{\prime}\left(t^{\prime}\right) h\left(s^{\prime}\right)\right) \geqslant 0,
\end{aligned}
$$


by Theorem (3.4)(a) $\rightarrow$ (d). By (d) $\rightarrow$ (a) of the same theorem, $\pi \pi^{\prime}$ is subnormal. (b) The case $S=S^{\prime}=N$ is stated in [11, Proposition 2, and 1, Lemma 7]. The proof in our general case is straightforward and will not be given here. Q.E.D.

Now we shall discuss quasinormality. To prove that each quasinormal operator is subnormal one usually constructs geometrically (matricially) a normal extension of a quasinormal operator (see, e.g., [5, Chapter 3, Proposition 1.7]). As a consequence of Lemma (3.8)(c) we obtain a geometry-free proof of this implication using only the positivity condition (HB), therefore quasinormality finds an appropriate place in our dilation theory picture of subnormality. We begin with a "lifting-of-thecommutant-free" proof of the following well-known result originally proved by Ito [9, Theorem 3], by using Bram's method.

(3.7) Proposition. Let $S$ be a semigroup with unit. Let $\pi: S \rightarrow B(H)$ be a semigroup homomorphism. If the operators $\pi(s), s \in S$, are commuting isometries, then $\pi$ is subnormal. If $\tau: S \rightarrow B(K)$ is the minimal normal extension of $\pi$, then the operators $\tau(s), s \in S$, are unitary.

Proof. By Theorem (3.4)(e) $\rightarrow(\mathrm{a})$, it is enough to show that if $T_{1}, \ldots, T_{n}$ are commuting isometries in $H$, then they are a subrormal $n$-tuple. For simplicity of notations let $n=2$. Let $x_{m n} \in H, m, n=0, \ldots, p, p \in N$. Then

$$
\sum_{m, n, j, k}\left(T_{1}^{m} T_{2}^{n} x_{j k}, T_{1}^{j} T_{2}^{k} x_{m n}\right)=\left\|\sum_{j, k=0}^{p} T_{1}^{p-j} T_{2}^{p-k} x_{j k}\right\|^{2} .
$$

One verifies this equality by grouping components of the second sum according to the order: $m<j, m \geqslant j, n<k, n \geqslant k$. Thus $T_{1}, T_{2}$ are a subnormal pair. To prove the second assertion one shows that each $\tau(s), s \in S$, is an isometry, by using the minimality condition. Q.E.D.

Let $T=V|T|$ be a polar decomposition of $T \in B(H)$ with $|T|=\left(T^{*} T\right)^{1 / 2}$, and an appropriate partial isometry $V$. To guarantee the uniqueness we shall always assume $\operatorname{ker} V=\operatorname{ker} T=\operatorname{ker}|T|$, and we shall refer to such a decomposition as the polar decomposition of $T$. $T$ is quasinormal if $T$ commutes with $T^{*} T$, or, equivalently, $|T|$ commutes with $V$.

(3.8) Lemma. (a) If $V \in B(H)$ is a quasinormal partial isometry, then ker $V$ reduces $V, V=V^{\prime} \oplus 0$ relative to the decomposition $H=(\operatorname{ker} V)^{\perp} \oplus \operatorname{ker} V$, and $V^{\prime}$ is an isometry.

(b) Let $T=V|T|$ be the polar decomposition of $T \in B(H)$. If $T$ is quasinormal, then $V$ is a quasinormal partial isometry, $V=V^{\prime} \oplus 0,|T|=P^{\prime} \oplus 0$ relative to the decomposition $H=(\operatorname{ker} T)^{\perp} \oplus \operatorname{ker} T, V^{\prime}$ is an isometry, $P^{\prime} \geqslant 0$, and $V^{\prime} P^{\prime}=P^{\prime} V^{\prime}$.

(c) Let $T=V|T|$ be the polar decomposition of $T \in B(H)$. Then $T$ is quasinormal if and only if $V,|T|$ is a subnormal pair.

Proof. (a) is clear, because $V^{*} V$ is the projection onto $(\operatorname{ker} V)^{\perp}$.

(b) Since $V|T|=|T| V$, and $V^{*} V$, being the projection onto the closure of the range of $|T|$, is a strong operator topology limit of polynomials in $|T|$, it follows that $V$ commutes with $V^{*} V$. 
(c) If $T$ is quasinormal, then, by (b), $V$ is a quasinormal partial isometry, which, by (a) and Proposition (3.7), is subnormal. By Corollary (3.6)(a), $|T|, V$ is a subnormal pair. The converse is obvious. Q.E.D.

(3.9) Lemma. (a) If $V, V^{\prime}$ are partial isometries which are jointly quasinormal, then $V, V^{\prime}$ are a subnormal pair.

(b) Let $T=V|T|, T^{\prime}=V^{\prime}\left|T^{\prime}\right|$ be the polar decompositions of $T, T^{\prime} \in B(H)$. Then $T, T^{\prime}$ are jointly quasinormal if and only if $|T|\left|T^{\prime}\right|=\left|T^{\prime}\right||T|, V V^{\prime}=V^{\prime} V, V\left|T^{\prime}\right|=$ $\left|T^{\prime}\right| V$, and $V^{\prime}|T|=|T| V^{\prime}$.

(c) If $T, T^{\prime}$ are jointly quasinormal, then $\left|T T^{\prime}\right|=|T|\left|T^{\prime}\right|, T T^{\prime}=V V^{\prime}|T|\left|T^{\prime}\right|$ is the polar decomposition of $T T^{\prime}$, and $V, V^{\prime}$ are jointly quasinormal.

Proof. Let $P=I-V^{*} V, P^{\prime}=I-V^{*} V^{\prime}$ be the projections onto $\operatorname{ker} V, \operatorname{ker} V^{\prime}$, resp. Then $P P^{\prime}=P^{\prime} P$ and both $P, P^{\prime}$ reduce both $V, V^{\prime}$. Consider the following decomposition of $H: I=\left(I-\left(P+P^{\prime}-P P^{\prime}\right)\right)+\left(P-P P^{\prime}\right)+\left(P^{\prime}-P P^{\prime}\right)+P P^{\prime}$. Since $P, P^{\prime}$ commute, $I-\left(P+P^{\prime}-P P^{\prime}\right)$ is the projection onto $\left\{P x+P^{\prime} y\right.$, $x, y \in H\}^{\perp}$, equal to $(\operatorname{ker} V)^{\perp} \cap\left(\operatorname{ker} V^{\prime}\right)^{\perp}$, and the above decomposition of $H$ is the orthogonal one. Relative to this decomposition, $V, V^{\prime}$ have form: $V=W \oplus 0 \oplus$ $U \oplus 0, V^{\prime}=W^{\prime} \oplus U^{\prime} \oplus 0 \oplus 0$. Now $W, W^{\prime}$ are commuting isometries, thus they are a subnormal pair, by Proposition (3.7), which proves (a). The proof of the remaining assertions is partly done in the first part of the proof of Theorem 4.5 of [10]. What is not done there, can be easily completed. Q.E.D.

Let $S$ be a semigroup with unit. Let $\pi: S \rightarrow B(H)$ be a semigroup homomorphism. For $s \in S$ let $\mu(s)=|\pi(s)|$ and let $\pi(s)=\theta(s) \mu(s)$ be the polar decomposition of $\pi(s)$. Define $\theta \mu$ on the product semigroup $S \times S$ by $\theta \mu(s, t)=\theta(s) \mu(t)$, $s, t \in S$.

(3.10) THEOREM. The following conditions are equivalent:

(a) $\pi$ is a quasinormal homomorphism.

(b) The operators in the family $\theta(S) \cup \mu(S)$ commute each other.

(c) $\theta \mu$ is a subnormal homomorphism.

(d) Each operator $\pi(s), s \in S$, is quasinormal and $\pi$ is a subnormal homomorphism.

Proof. (a) $\rightarrow$ (b) follows from Lemma (3.9)(b).

(b) $\rightarrow$ (c) By Lemma (3.9)(c), $\theta$ and $\mu$ are semigroup homomorphisms, and for each $n \in N, s_{1}, \ldots, s_{n} \in S$ the partial isometries $\theta\left(s_{1}\right), \ldots, \theta\left(s_{n}\right)$ are jointly quasinormal. Hence they are a subnormal $n$-tuple, by Lemma (3.9)(a). By Theorem $(3.4)(\mathrm{e}) \rightarrow(\mathrm{a}), \theta$ is a subnormal homomorphism. Now (b) follows from Corollary (3.6)(a), because $\mu$ is normal.

(c) $\rightarrow$ (d) Since $\pi(s)=\theta \mu(s, s), \pi$ is subnormal. Since $\theta \mu$ is a semigroup homomorphism, $\theta(s) \mu(s)=\theta \mu((1, s)(s, 1))=\theta \mu(1, s) \theta \mu(s, 1)=\mu(s) \theta(s), s \in S$. Thus $\pi(s)$ is quasinormal, $s \in S$.

(d) $\rightarrow$ (a) Fix $s, s^{\prime} \in S$. The pair $T=\pi(s), T^{\prime}=\pi\left(s^{\prime}\right)$ is subnormal. By Corollary (3.6)(b), if $N \in B(K)$ is the minimal normal extension of $T$, then there is $T^{\prime \prime} \in B(K)$ extending $T^{\prime}$ and commuting with $N$, hence with $N^{*} N$. Since $T$ is quasinormal, $H$ 
reduces $N^{*} N$ and $N^{*} N \mid H=T^{*} T$, by Theorem 2 of [15], whose "lifting-of-thecommutant-free" proof can be given by using Lemma (3.8)(b). Therefore $T^{\prime}$ commutes with $T^{*} T$. Q.E.D.

The implication (a) $\rightarrow(d)$ is Theorem 4.5 of [10]. The equivalence of (b) and (d) for $S=N^{2}$ is Theorem 5 of [15].

\section{REFERENCES}

1. M. B. Abrahamse, Commuting subnormal operators, Illinois J. Math. 22 (1978), 171-176.

2. T. Ando and W. Szymanski, Order structure and the Lebesgue decomposition of positive definite operator functions, Indiana Univ. Math. J. 35 (1986), 157-173.

3. A. Athavale, Holomorphic kernels and commuting operators, preprint, 1986.

4. J. Bram, Subnormal operators, Duke Math. J. 22 (1955), 75-94.

5. J. B. Conway, Subnormal operators, Pitman, Boston, Mass., 1981.

6. J. B. Conway and W. Szymanski, Linear combinations of hyponormal operators, Rocky Mountain J. Math. (to appear).

7. M. Embry, A generalization of the Halmos-Bram criterion for subnormality, Acta Sci. Math. (Szeged) 35 (1973), 61-64.

8. P. R. Halmos, Normal dilations and extensions of operators, Summa Brasil. Math. 2 (1950), 125-134.

9. T. Ito, On the commutative family of subnormal operators, J. Fac. Sci. Hokkaido Univ. (Sapporo) 14 (1958), 1-15.

10. A. Lubin, Weighted shifts and commuting normal extensions, J. Austral. Math. Soc. Ser. A 27 (1979), 17-26.

11. M. Slocinski, Normal extensions of commutative subnormal operators, Studia Math. 54 (1976), 259-266.

12. F. H. Szafraniec, Dilations on involution semigroups, Proc. Amer. Math: Soc. 66 (1977), 30-32.

13. B. Sz.-Nagy, Extensions of linear transformations in Hilbert space which extend beyond the space, Appendix to F. Riesz and B. Sz.-Nagy, Functional Analysis, Ungar, New York, 1960.

14. W. Szymanski, Positive forms and dilations, Trans. Amer. Math. Soc. 301 (1987), 761-780.

15. T. Yoshino, On the commuting extensions of nearly normal operators, Tôhoku Math. J. 25 (1973), $263-272$.

Department of Mathematical Sciences, West Chester University, West Chester, PennSYI.VANIA 19383 